\title{
Contabilidad, economía de la cultura y sectores creativos: necesidades y desafíos de una vinculación técnica y disciplinar*
}

\author{
Accounting, cultural economics and creative sectors: Needs and challenges of a technical and disciplinary \\ linkage \\ Contabilidade, economia da cultura e setores criativos: necessidades e desafios de um vínculo técnico e \\ disciplinar
}

Juan David Arias Suárez ${ }^{\text {a }}$

Politécnico Grancolombiano, Colombia

DOI: https://doi.org/10.11144/Javeriana.cc22.cecs

jdarias@poligran.edu.co

ORCID: https://orcid.org/0000-0002-4218-953X

Recibido: 05/03/2020

Carolina Asuaga

Aceptado: 15/10/2021

Universidad de la República, Uruguay

Publicado: 31/12/2021

ORCID: https://orcid.org/0000-0002-4454-4152

Vanessa Cano Mejía

Institución Universitaria de Envigado, Colombia

ORCID: https://orcid.org/0000-0001-6084-668X

\section{Resumen:}

Este artículo reflexiona y busca llamar la atención de la comunidad académica sobre las urgentes necesidades conceptuales, técnicas y normativas de co-construir una contabilidad que represente realidades diversas y reconozca el amplio contexto social de las prácticas contables. Se hace un análisis socioeconómico de la transformación de los modelos de producción y de negocios, contemporáneamente vinculados a estructuras de economías emergentes como industrias y servicios culturales y creativos; lo cultural, lo simbólico y lo creativo representan el futuro de las industrias, los cuales se aglutinan en el estudio de la economía de la cultura, enfocada en el patrimonio cultural, las artes escénicas y la industria cultural, donde lo intangible y los nuevos modelos de negocios retan a los saberes contables y económico-financieros. Se reflexiona sobre los desafíos del control y la representación contables frente a productos, servicios y estructuras organizacionales y comerciales, que no siguen los cánones habituales de la existencia material y el intercambio económico. La necesidad de una contabilidad sociocultural que controle, represente y de cuenta de las nuevas realidades económico-culturales, establece desafíos de una vinculación técnica y disciplinar de la contabilidad con la economía de la cultura.

Códigos JEL: L19, M41, Z10.

Palabras clave: Economía de la cultura, contabilidad, representación contable, sectores creativos.

\section{Abstract:}

This paper reflects and seeks to draw the attention of the academic community to the urgent conceptual, technical and normative needs to co-construct an accounting that represents diverse realities and recognizes the broad social context of accounting practices. A socioeconomic analysis of the transformation of production and business models is presented, contemporaneously linked to structures in emerging economies such as cultural and creative industries and services; the cultural, the symbolic and the creative represent the future of industries, which are brought together in the study of the economy of culture, focused on cultural heritage, the performing arts and the cultural industry, where the intangible and new models businesses challenge accounting and economicfinancial knowledge. It reflects on the challenges of accounting control and representation in the face of products, services and organizational and commercial structures, which do not follow the usual canons of material existence and economic exchange. The need for a sociocultural accounting that controls, represents and accounts for the new economic-cultural realities, establishes challenges of a technical and disciplinary link between accounting and the economy of culture.

JEL Codes: L19, M41, Z10.

Keywords: Cultural economy, accounting, accounting representation, creative sectors.

Notas de autor

\footnotetext{
a Autor de correspondencia.E-mail: jdarias@poligran.edu.co
} 


\section{Resumo:}

Este artigo reflete e busca chamar a atenção da comunidade acadêmica para a urgente necessidade conceitual, técnica e normativa de co-construir uma contabilidade que represente realidades diversas e reconheça o amplo contexto social das práticas contábeis. Faz-se uma análise socioeconômica da transformação dos modelos produtivos e de negócios, contemporaneamente vinculados a estruturas de economias emergentes, como indústrias e serviços culturais e criativos; o cultural, o simbólico e o criativo representam o futuro das indústrias, que se articulam no estudo da economia da cultura, focada no patrimônio cultural, nas artes cênicas e na indústria cultural, onde o intangível e os novos modelos de negócios desafiam os conhecimentos contábeis e econômico-financeiros. Refletese sobre os desafios do controle e representação contábeis frente aos produtos, serviços e estruturas organizacionais e comerciais, que não seguem os cânones usuais da existência material e do intercâmbio econômico. A necessidade de uma contabilidade sociocultural que controle, represente e dê conta das novas realidades econômico-culturais, estabelece os desafios de um vínculo técnico e disciplinar entre a contabilidade e a economia da cultura.

Códigos JEL: L19, M41, Z10.

Palavras-chave: Economia da cultura, contabilidade, representação contábil, setores criativos.

\section{Introducción}

Las transformaciones sociohistóricas, culturales, políticas, económicas y biosféricas de la vida en el planeta son cada vez más complejas e inciertas. Los mercados y las manifestaciones humanas son constantemente dinamizados e influenciados por la concentración y transfronterización de capitales forjando nuevas interpretaciones y articulaciones de las relaciones entre las organizaciones, el Estado, la sociedad y el ambiente. Esto reta a los saberes a comprender el pluriverso de posibilidades de organización de las industrias, las actividades socioculturales y la creatividad de las mismas, que cada vez son menos rígidas y más líquidas (Bauman, 2013).

En ese contexto emerge la economía de la cultura como un constructo que comprende las relaciones económicas de las actividades, bienes y servicios culturales, lo cual desafía a un plexo importante de profesionales, organizaciones y disciplinas para lograr que lo cultural no se erija como un medio adicional de crematística atomizado en la industrialización. Los desarrollos, mutaciones e innovaciones en la producción y comercialización de bienes culturales y en la prestación de servicios asociados a la cultura han generado sectores emergentes, creativos y/o innovadores que actúan por medio de nuevas formas e instrumentos de intercambio, financiación y acumulación de capital, transformando las estructuras económicas, institucionales y socio-organizacionales que tradicionalmente ha representado la contabilidad.

El estudio de las implicaciones económicas en las nuevas formas de producción se puede conocer desde las disciplinas como la economía creativa ${ }^{1}$, que según las estimaciones oficiales es la responsable de más del 6\% de la economía mundial, y en la que diversos países como China, India, la Unión Europea y los EEUU están apostando fuertemente (Rausell-Köster, 2017). De hecho, el Banco Interamericano de Desarrollo (BID) desde hace más de una década ha mostrado una estrategia que apuesta a las industrias y servicios creativos como motor de desarrollo económico de América Latina y el Caribe (Quartesan, Romis, \& Lanzafam, 2007; BID, 2009; Benavente \& Grazzi, 2017; Luzardo, De Jesús, \& Perez, 2017, Finlev, Maguire, Oppenheim et al., 2017). Hasta 2012, la UNESCO sostenía que estas disciplinas han crecido 2,5 veces más que el promedio de los demás sectores de la economía a nivel mundial. Su incidencia en el desarrollo económico es notable. Diversos autores sustentan los impactos positivos y los efectivos multiplicadores en empleo y activación de la economía que genera la economía de la cultura (Bocella \& Salerno, 2016).

Asimismo, no es posible obviar la tecnología en el contexto de la industria y los sectores creativos. Los avances tecnológicos fueron parte de la discusión seminal en el desarrollo de la economía de la cultura, disciplina de la economía aplicada y precursora de la economía creativa. En los últimos años, con las transformaciones del ámbito cultural, espacios de consumo cultural se han visto fortalecidos con las 
plataformas tecnológicas y las redes sociales, generando nuevos modelos de negocios e industrias exitosas como Netflix en audiovisual y Spotify en el consumo de música.

Desde los planteamientos de Araújo (1998) es clave comprender que, si el contexto socioeconómico cambia, la contabilidad también lo debe hacer para representar la realidad con mayor pertinencia. Por ello es clave ubicar a la contabilidad más allá de una técnica instrumental y registral, pensándola como un saber disciplinar social encargada de estudiar hechos y problemas socioeconómicos como el control integral de la riqueza (Sarmiento, 2007) y la representación integral de la realidad social (Arias, 2017; Arias \& Cano, 2020). Esta mirada amplia de la contabilidad, abiertamente multiparadigmática e interdisciplinar permite fortalecer los márgenes de la contabilidad (Miller, 1998) en función de la relación contabilidad-economía de la cultura. Así, el saber contable es retado y entra en tensión para avanzar en forjar un corpus académico y técnico que tenga mayor pertinencia para estudiar los procesos de reconocimiento, medición, valoración, consolidación, control, planificación, representación y revelación de hechos socioeconómicos como los derivados económicos de la cultura.

En igual sentido, las perspectivas que ubican a la contabilidad como práctica social e institucional (Hopwood \& Miller, 1994; Potter, 2005) aportan a la comprensión de las múltiples manifestaciones instrumentales y de procesos de control que se desarrollan en el ámbito cultural, potenciando una contabilidad más cercana a la cotidianidad social.

Desde hace varias décadas se ha intentado determinar las limitaciones y dificultades de la contabilidad para estudiar procesos asociados a lo cultural (Carnegie \& Wolnizer, 1995, 1996, 1999; Micallef \& Peirson, 1997; Mautz, 1988), pero se termina desarrollando nociones y técnicas ficticias que no reconocen adecuadamente la realidad. Estas perspectivas del saber contable implican elaborar un constructo epistémico más robusto, para lo cual se sugiere estudiar autores latinoamericanos como Sá (1995), García-Casella (2012), Sarmiento (2007), Gómez (2011), Ospina (2006), Gil (2004) y Rueda (2002), identificando, además, puntos clave para fortalecer el saber.

En este orden de ideas este artículo problematiza y parte de interrogantes como ¿El clásico método veneciano de contabilidad por partida doble está vigente y es pertinente para la representación de la actividad e industria cultural? ¿El valor razonable y las métricas emanadas del International Accounting Standards Board (IASB) son suficientes para ilustrar los crecimientos, fluctuaciones y decrecimientos de las actividades diversas? ¿Son comparables los resultados financieros y no-financieros de las organizaciones y estructuras emanadas de la cultura contemporánea?

Los estudios previos sobre contabilidad y procesos culturales han hecho énfasis en la propagación de datos desproporcionados entre las variables de stock, de flow, en la centralización del discurso contable en los activos, y en procesos lineales de depreciación y deterioro de valor (Zan, Blackstock, Cerutti et al., 2000) que no son propiamente coherentes con las nuevas formas de la economía de la cultura. Algunas investigaciones sobre estos tópicos se encuentran en Cieslewicz (2014), Chanchani \& MacGregor (1999), Gray (1988), Braun \& Rodriguez (2008), Chenhall (2003) y Harrison \& McKinnon (1999). Por ello Jeacle (2012) y Jeacle \& Miller (2016) insisten en las pocas bases contables para abarcar fuerzas sociales significativas (rituales, tradiciones y acciones de tradición) que dominan la vida cultural y le dan sentido a la lógica económica.

Espacios de interacción sociocultural como TriAdvisor, Facebook, Twitter, Netflix, Spotify, Youtube, Instagram, Whatsapp, Google, los mass media y los medios digitales y cibernéticos como epicentros de la comunicación mundial, son algunas muestras de cómo las nuevas industrias reconfiguran las acciones humanas hacia entornos virtuales y de plataformas tecnológicas. Estas manifestaciones de la vida cultural hacen parte del campo de estudio de la economía de la cultura, que insertas en el campo financiero, deben ser representadas por la contabilidad como infraestructura captadora del flujo de la riqueza, buscando representar su pluralidad económica y su singularidad simbólica. Empero ¿está realmente preparada la contabilidad para controlar, representar y dar cuenta de las nuevas estructuras de los negocios y su captación de valor? 
Este documento de reflexión busca avanzar en los fundamentos académicos que permitan a la contabilidad representar dimensiones complejas como la cultura (Cano, 2018; Cano, Arias \& Asuaga, 2020).

\section{Antecedentes sociohistóricos de la economía de la cultura}

Desde hace más de un siglo los aspectos económicos de la cultura han sido tema de interés para algunos economistas como Kindermann (1903) y Drey (1910). A su vez, la aplicación de herramientas propias de la ciencia económica al estudio de los costos de producción y a la productividad de los bienes y servicios culturales tienen su origen en la obra de Baumol \& Bowen (1966) Performing Arts-The Economic Dilemma, investigación solicitada por el sector teatral de Broadway a raíz de la crisis que estaban afrontando. En el informe presentado, los autores dividen la producción cultural en dos sectores: el compuesto por las "artes escénicas y musicales ejecutadas en vivo (performing arts), donde los autores sostienen que no es posible incorporar tecnología que permita bajar costos y consecuentemente aumentar la productividad, y otro sector, el tradicional, que sí lo permite" (Asuaga \& Esmoris, 2006, p. 3).

A partir de la obra de Baumol \& Bowen (1966) no ha cesado la discusión acerca de los elementos de la vida económica que se deben considerar como parte del estudio de la economía de la cultura. La inserción de los aspectos tecnológicos o las artes escénicas han sido algunos de los aspectos más polémicos. En los últimos dos lustros se ha hecho hincapié en que "la denominada economía cultural, y las industrias culturales y creativas son factores que impulsan el crecimiento económico" (Bocella \& Salerno, 2016, p. 291). A pesar de múltiples ganancias para algunos subsectores aún se discuten las formas en que se financian negocios cinematográficos, los museos y las actividades de las artes escénicas, generando todo tipo de debates y fomentando la búsqueda de recaudaciones externas, colectas públicas o patrocinios.

Este documento defiende que la Economía de la Cultura es una rama de la economía aplicada, lo cual provoca diferentes reacciones, dando lugar a que tópicos usuales que eran entendidos potestad de la economía pública, pasan a ser analizados por esta nueva rama de la economía que intenta dar respuesta a la pertinencia conceptual de la financiación estatal. Parte de los detractores de la teoría presentada se basan en los avances tecnológicos que ya existían en esos años en los recitales de rock, que abarcaban un gran número de público mediante el uso de tecnología en el audio, y la reproducción por una relativamente reciente televisión por aire, aspectos inimaginables décadas atrás, empero, estos son fenómenos culturales que tienen dimensiones sociales y simbólicas más allá de la organización de un evento musical; las ideas y representaciones culturales del fenómeno artístico son las que movilizan la estructura económica de la industria y sus formas de operación.

No obstante, aún ante las numerosas críticas, la idea central había sido aceptada por la doctrina mayoritaria (Throsby \& Withers, 1979; Frey \& Pommerehne 1989; Throsby 1994), y la economía de la cultura, como nueva disciplina, ha contado con numerosos aportes. Diversos autores, como los suizos Frey \& Stutzer (2002), la francesa Benhamou (2002) el italiano Mossetto (2013), el australiano Throsby (1979; 1994), los españoles Bonet (2003) y Rausell-Köster (1999), entre otros, han contribuido a la consolidación de la Economía de la Cultura como una subdisciplina dentro de la Economía que trata de aproximarse a los fenómenos de la creación, producción, distribución y consumo de los bienes y servicios culturales.

En este sentido, es menester comprender que "el éxito económico es secundario al valor simbólico, en la competencia que se da por el capital cultural en la forma de reconocimiento, reputación y legitimidad" (Anheier, Gerhards \& Romo, 1995, p. 963). Esto es importante para el análisis contextual, ya que en muchas ocasiones se toma al sector cultural y creativo como otra forma de generar lucro, y ello puede sepultar la mística sociohistórica de la cultura, cuando el valor simbólico per se, puede conseguir el objetivo industrial, y el papel del profesional debe ser su representación, protección y equilibrio.

A su vez, la economía de la cultura ha hecho importantes avances en el estudio de las relaciones económicas vinculadas a la cultura (Palma \& Aguado, 2010; Aguado, Palma \& Pulido, 2017). Asimismo, es importante 
atender las reflexiones de Shorthose (2004), cuando comenta la importancia de hacer ejercicios etnográficos antes de medir los impactos del sector cultural en los desarrollos industriales y creativos ya que podrían ser mediciones parciales por no conocer las características culturales más singulares, quizá ocultas, temporales y espontáneas. En la tabla 1 se sintetizan los grandes objetos de estudios de la economía de la cultura.

TABLA 1

Taxonomías del análisis económico aplicado a la cultura

\begin{tabular}{|c|c|c|c|}
\hline $\begin{array}{l}\text { Vertiente } \\
\text { económica }\end{array}$ & $\begin{array}{l}\text { Ámbito } \\
\text { concreto }\end{array}$ & $\begin{array}{c}\text { Temáticas de interés } \\
\text { particular }\end{array}$ & Temas transversales \\
\hline \multirow{5}{*}{$\begin{array}{l}\text { Economía } \\
\text { de la } \\
\text { cultura }\end{array}$} & Artes escénicas & Asistencia y demanda & \multirow{4}{*}{$\begin{array}{l}\text { Enfermedad de los costos. } \\
\text { Formación de gustos y } \\
\text { hábitos. Adicción racional. } \\
\text { Aprendizaje a través del } \\
\text { consumo. Capital cultural. } \\
\text { Valor cultural. Formas } \\
\text { organizacionales y de } \\
\text { gestión de las instituciones. } \\
\text { Organizaciones con y sin } \\
\text { ánimo de lucro. } \\
\text { Organizaciones estatales. } \\
\text { Comercio internacional de } \\
\text { bienes culturales. } \\
\text { Estadísticas del sector } \\
\text { cultural. }\end{array}$} \\
\hline & Artes visuales & $\begin{array}{l}\text { Demanda por razones } \\
\text { estéticas o como activos de } \\
\text { instrumentos financieros. } \\
\text { Subasta y precio de obras } \\
\text { de arte. }\end{array}$ & \\
\hline & $\begin{array}{l}\text { Patrimonio } \\
\text { histórico y } \\
\text { construido }\end{array}$ & $\begin{array}{l}\text { Valoración, control y } \\
\text { conservación. Economía } \\
\text { del patrimonio. }\end{array}$ & \\
\hline & $\begin{array}{l}\text { Industrias } \\
\text { culturales }\end{array}$ & $\begin{array}{l}\text { Organización industrial, } \\
\text { localización, clúster, distrito } \\
\text { cultural. Economía del } \\
\text { libro, del cine, del arte. }\end{array}$ & \\
\hline & Política cultural & $\begin{array}{l}\text { Por qué, de qué forma y en } \\
\text { qué medida debe intervenir } \\
\text { el Estado de los mercados y } \\
\text { ámbitos culturales. }\end{array}$ & $\begin{array}{l}\text { Financiación pública de la } \\
\text { cultura, mecenazgo, precio } \\
\text { fijo del libro, derechos de } \\
\text { autor. }\end{array}$ \\
\hline \multirow[b]{2}{*}{$\begin{array}{l}\text { Economía } \\
\text { creativa }\end{array}$} & $\begin{array}{l}\text { Industrias } \\
\text { creativas }\end{array}$ & $\begin{array}{l}\text { La cultura como factor de } \\
\text { innovación, publicidad, } \\
\text { diseño gráfico, moda, } \\
\text { arquitectura, videojuegos, } \\
\text { software. }\end{array}$ & $\begin{array}{l}\text { Copyright, ciudades } \\
\text { creativas, clase creativa }\end{array}$ \\
\hline & Creatividad & $\begin{array}{l}\text { Innovación, productividad, } \\
\text { crecimiento económico. El } \\
\text { crecimiento económico } \\
\text { llega con la innovación, } \\
\text { cuya base está en la } \\
\text { estimulación creativa de la } \\
\text { densiddad cultural. }\end{array}$ & $\begin{array}{l}\text { Formas de producción y } \\
\text { estimulación de la } \\
\text { creatividad. } \\
\begin{array}{l}\text { Esquemas cognitivos de la } \\
\text { creatividad }\end{array}\end{array}$ \\
\hline
\end{tabular}

Fuente: adaptación de Herrero (2002) y Palma \& Aguado (2010).

Estas temáticas se han desarrollado para las diversas actividades culturales, surgiendo, entre otros, estudios relativos a la economía del patrimonio, economía del teatro o economía del museo. En cuanto a los sectores objeto de estudio de lo que tradicionalmente se denomina economía de la cultura, múltiples referencias se enfocan en tres grandes divisiones:

Patrimonio cultural. En este ítem se incluye la identificación simbólica en común que tiene un determinado territorio, la que puede expresarse por medio de expresiones tangibles e intangibles. Es por eso que abarca, en una enumeración no taxativa, al patrimonio histórico monumental, pero también contempla la gastronomía típica, los deportes declarados de interés patrimonial, la música que da identidad a un territorio, las festividades típicas, los museos y el turismo cultural, entre otros.

Artes escénicas. Este sector contempla las expresiones artísticas en vivo, tales como conciertos, tanto de música clásica como popular, teatro, danza, ópera y circo, entre otras.

Industria cultural. Se refiere a la música grabada, la industria editorial y cinematográfica. Tienen en común la existencia de un master, que luego es reproducido en forma industrial en un proceso similar a la de la industria no cultural. Además, "la definición de la industria cultural puede incluir la música, las industrias relacionadas con el arte, la escritura, la moda y el diseño, los medios de comunicación y la producción artesanal" (Bocella \& Salerno, 2016, p. 292).

Aunque existió un amplio consenso académico para determinar los tres sectores anteriores, existieron también discusiones sobre si la televisión y la radio eran objeto de estudio de la economía de la cultura o debían circunscribirse a otra rama de la economía aplicada como es la economía de la comunicación. Sin embargo, la discusión fue zanjada y tanto la radio como la televisión, sea por aire o por abonados, forman parte de la industria cultural independientemente del contenido de la programación. No obstante, no existió consenso con el sector publicitario, y la corriente principal de economía de la cultura no incluye a la publicidad como objeto de estudio. 


\section{Lo cultural: aproximaciones a la economía y sector creativo}

A inicios del siglo XXI surge el concepto de economía creativa (Howkins, 2002) donde las ideas constituyen los principales aportes y resultados. Para la UNESCO las industrias culturales y creativas son "aquellos sectores de actividad organizada que tienen como objeto principal la producción o la reproducción, la promoción, la difusión y/o la comercialización de bienes, servicios y actividades de contenido cultural, artístico o patrimonial" (UNESCO, 2013, párr. 5). Para las Naciones Unidas las industrias culturales y creativas combinan la creación, producción y comercialización de contenidos creativos que sean intangibles y de naturaleza cultural. Estos contenidos están normalmente protegidos por el derecho de autor y pueden tomar la forma de un bien o servicio. Incluyen además toda producción artística o cultural, la arquitectura y la publicidad. Por su amplitud no existe consenso en los sectores que componen este sector.

Tomando los referentes de la UNESCO y PNUD (2014), existen varios sistemas de clasificación de este tipo de industrias culturales y creativas: a) Modelo DCMS, b) modelo de textos simbólicos, c) modelo de círculos concéntricos, d) modelo de la OMPI, e) modelo del instituto de estadísticas de la UNESCO, y f) modelo de americanos por las artes. Estos subsistemas de clasificación tienen múltiples puntos de coincidencia, mostrando la diversidad, divergencias y convergencias que coexisten en el sector.

Según el BID (2017), la industria creativa generó 1,9 millones de puestos de trabajo en América Latina y el Caribe, e ingresos por más de 124 mil millones de dólares. Cabe cuestionarse qué es lo que atrae tanto la atención de las organizaciones internacionales, las que encuentran en este sector una forma de desarrollo económico para América Latina y el Caribe.

Los sectores culturales y creativos representan casi el 4,5\% de la economía europea, gracias a que casi 1,4 millones de pequeñas y medianas empresas generan y distribuyen contenido creativo en toda Europa. Los sectores culturales y creativos han demostrado una gran capacidad de recuperación durante la crisis (en realidad continuaron creciendo) al tiempo que estimulaban la creatividad y la innovación en otros sectores. Alrededor de 8,5 millones de personas trabajan en sectores creativos en toda Europa, y muchos más si tenemos en cuenta su impacto en otros sectores como el turismo y la tecnología de la información. (European Creative Business Network, 2015, p. 6)

Lo anterior puede entenderse caracterizando el desarrollo de este sector en un sector dinámico, esto es, un sector que crece por encima de la media de la economía. Los emprendimientos dinámicos poseen ciertas características en común, como por ejemplo que son creados por emprendedores jóvenes (entre 25 y 35 años), que han tenido pasaje por estudios universitarios. El personal también es joven, con estudios superiores a la media, utilizan las tecnologías, reciben un mejor salario y muestran mayor satisfacción, lo que conlleva a una productividad más elevada que la que se da en sectores no-creativos.

Es importante para el desarrollo de este tipo de emprendimientos el entorno económico, destacándose Estados Unidos y Europa como las regiones más favorables para el éxito de los emprendimientos dinámicos. En América Latina se destaca Chile y lo siguen Brasil, México, Uruguay y Colombia como los países con mejor ecosistema emprendedor (Kantis, Federico, \& Ibarra, 2016). Asimismo, si el sector creativo se combina con el tecnológico surgen emprendimientos altamente dinámicos, como por ejemplo Netflix, Spotify, Facebook, WhatsApp. Estos impulsan a la economía en su conjunto, de ahí el interés de gobiernos y organizaciones en fomentar este tipo de emprendimientos.

Cabe destacar la diferencia conceptual entre talento, creatividad e innovación. Si por ejemplo se compone una canción, puede ser un producto creativo, pero el compositor no necesariamente tiene el talento para interpretarlo. Asimismo, el intérprete, que puede ser un destacado concertista, no necesariamente es un sujeto creativo. La creatividad potencia, estimula y favorece a la innovación, pero no todo proceso creativo es innovador. Una obra creativa no implica necesariamente innovación. Son características distintas, singulares, las cuales permite conocer cómo son las formas de construcción y extracción de valor social, económico y simbólico que representa la contabilidad. 


\section{Desafíos para la investigación y representación contables}

Aunque Smith (1776) ya teorizaba sobre el rol de los artistas en la contribución a las riquezas de las naciones, tanto economistas como contadores y administradores han tenido resistencia para comenzar a analizar el sector creativo con su herramental metodológico. Las ciencias económico-financieras perennemente han estado dominadas por la racionalidad crematística, dejando de lado el análisis profundo de los sectores que no representen impacto en la economía. Por tanto, es necesario "comprender la manera como actúan el capital simbólico y los mecanismos de planeación y gestión, en la expansión de nuevos campos y en la colonización del campo cultural por el campo económico y de los negocios" (Gómez \& Ospina, 2009, p. 33). La epistemología de la contabilidad es retada en su núcleo técnico y conceptual para moldear su andamiaje y representar las nuevas formas de la industria. Las necesidades y desafíos de la vinculación representada en la figura 1 ejercen presión para dar cuenta del devenir organizacional.

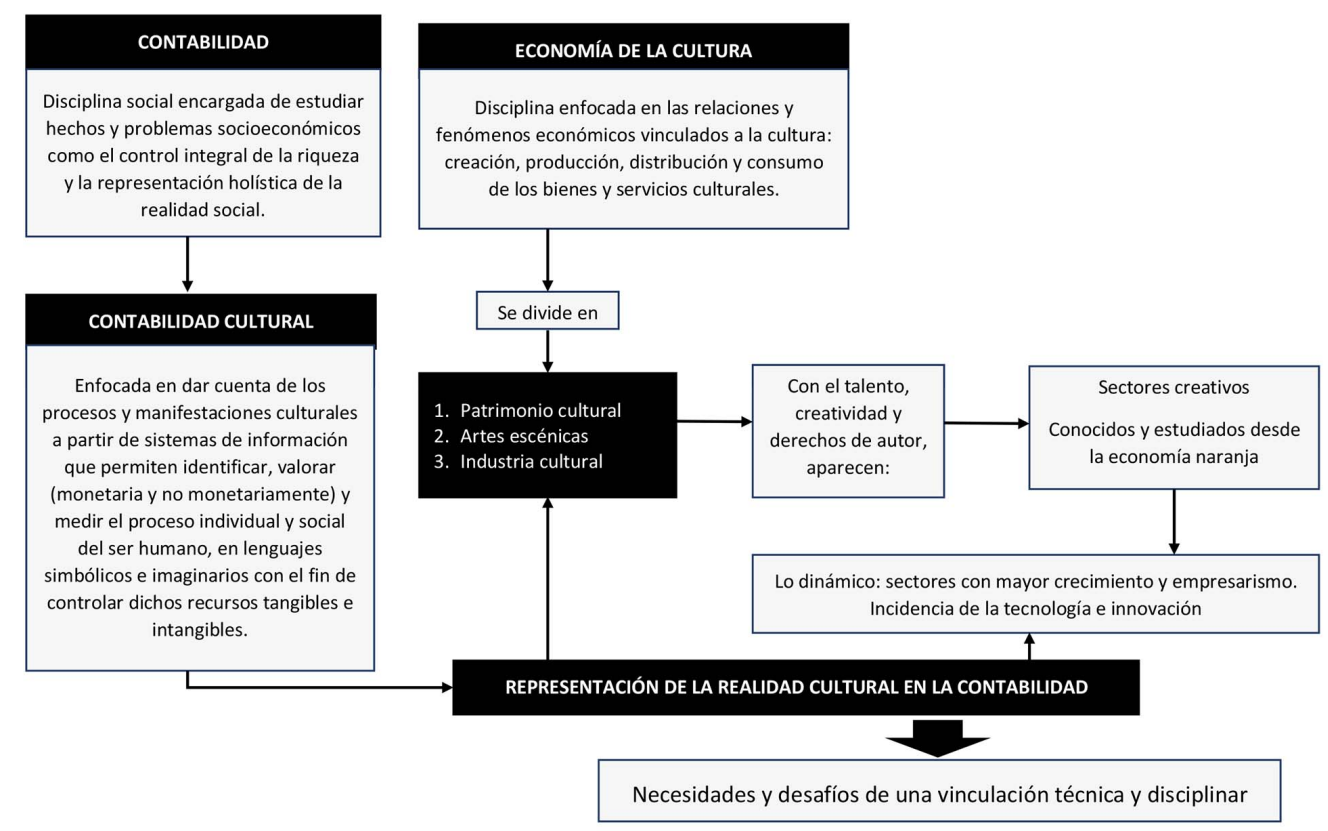

FIGURA 1

Vinculación disciplinar entre la contabilidad y la economía de la cultura

Fuente: elaboración propia.

Shorthose (2004, p. 150) indicó que la contabilidad tenía dificultades para representar la economía de la cultura debido a su estructura: trabajadores independientes, microempresas, grupos informales, empleados temporales y gran contenido intangible; su perspectiva mencionaba la necesidad de un estudio etnográfico previo a la representación contable. En el espectro organizacional, Pratt \& Jeffcutt (2011), relacionan las características de las industrias culturales, así:

- Una estructura industrial mejor caracterizada como ecosistema.

- Redes superpuestas, mediadas por intermediarios y facilitadas por el movimiento de trabajadores independientes y la interacción informal.

- Falta relativa de empresas medianas.

- Preponderancia de compañías basadas en proyectos de corta duración con trabajadores independientes.

- Incertidumbre masiva del mercado, junto con un riesgo muy alto de fracaso.

- Rotación muy rápida de la innovación, la moda y el ciclo del producto.

- Un sistema 'ganador se lleva todo': diferencias masivas entre lo que significa ganar y perder. 
- Tensión entre regulación de contenidos y regulación de la competencia.

En este contexto es menester reflexionar acerca de ¿cómo son representadas estas características en un balance contable? ¿Cómo operan los sistemas de control y rendición de cuentas en modelos de negocios nolineales ni progresivos económicamente?

Por ejemplo, los productos y los servicios de las industrias y servicios culturales y creativos no siempre son constantes ni progresivos, tienden a ser cíclicos o de producción única, por tanto, la comparación entre periodos contables y los registros históricos no son medidas eficientes para la proyección de un escenario contable futuro. La valuación de activos y bienes patrimoniales inmateriales, históricos, materiales (arquitectónicos, urbanos, arqueológicos), naturales o mixtos, implican mediciones y valoraciones que integren valores sociales, formales, simbólicos, de apropiación, de reconstrucción y de uso, y no sólo costo histórico o valor razonable. "La contabilidad en esta vía logra comprender la dinámica económica de circulación de la riqueza y también alcanza a consolidar en cuentas, balances y múltiples formas de representación, la interacción social" (Arias \& Cano, 2018, P. 39).

Desde lo normativo, el modelo de información financiera y de contabilidad internacional tiene escasos lineamientos técnicos específicos o procedimentales para abarcar los bienes y servicios culturales, a pesar de que "los estudios de métrica relativos a las industrias culturales y creativas son clave en la elaboración de políticas públicas culturales que colaboren en el fomento de la diversidad cultural” (Fuertes \& Badillo, 2016, p. 64). Quizá por ello, en las legislaciones nacionales se pueden encontrar algunas aproximaciones en lo referente a contabilidad pública e inventario de activos sociales o culturales, pero aún no existen marcos técnicos o principios contables para medir y valorar el plexo de productos derivados del sector cultural.

Por ejemplo, en la norma internacional de contabilidad 38 (activos intangibles), se pueden establecer algunas bases para representar productos culturales como las películas, pero pululan las condiciones para su reconocimiento como activo intangible, debido a la fiabilidad de su costo de valoración y su probabilidad de generar beneficios económicos futuros, aunado a su valor razonable, cálculo de deterioro de valor y determinación de la vida útil. La norma está concebida para reconocer lo que razonablemente genere utilidad monetaria de acuerdo con el modelo de producción tradicional, por tanto, un bien o servicio cultural cuyo origen, producción, distribución y consumo son complejos, termina siendo reconocido y valorado de forma simple y reductiva, lo cual no es una representación 'fiel' de su realidad económica. De hecho, la solución normativa es llegar a 'la mejor estimación posible', lo cual muchas veces termina desestimando la cultura por la miopía de las técnicas.

Lo mismo ocurre con la valoración y revaloración de inventarios para bienes culturales en función de la Norma Internacional de Contabilidad 2, donde se requiere utilizar el método de identificación específica de costos individuales, ya que los productos artísticos y/o artesanales normalmente son únicos. No obstante, para productos como una obra teatral o una película cuya reproducción no genera ingresos causales inmediatos, sino una distribución de ingresos de largo plazo en función de su visualización en el tiempo, con valores decrecientes a razón de la pérdida de interés del público (una especie de desmantelamiento), requiere mediciones complejas que puedan representar la realidad económica de estas actividades. Es importante tener presente que estas formas de acumulación de valor pueden verse afectadas con contratos específicos donde solo se negocie el derecho de reproducir y no el derecho intelectual de la obra.

Igualmente ocurre con el valor neto de realización, donde los precios estimados de venta guardan un valor relativo dependiendo del arraigo cultural o la especulación simbólica. La lógica financiera de mercado mercantiliza cualquier tipo de bien o servicio y establece precios, pero no todo funciona igual con las industrias y servicios culturales y creativos. En esa vía, coexisten reclamos, denuncias y señalamientos sobre la mercantilización de la cultura por el actual sistema de financiarización. "En efecto, dicho sistema genera valoraciones financieras que se presentan como equivalentes de la cultura y no coadyuvan a su protección histórica, al resguardo de su identidad y a su adecuada apropiación social” (Cano, 2018, pp. 111-112). 
En este sentido, la contabilidad está en capacidad de generar respuestas alternativas a los modelos financieros de medición de la producción, los servicios y las organizaciones, tejiendo mixturas disciplinares para dar respuesta a la realidad. Así, un saber contable social que elabore sistemas de información, control, representación y rendición de cuentas sobre hechos y fenómenos individuales y colectivos, tangibles e intangibles que evidencien la realidad cultural y simbólica puede denominarse contabilidad cultural y fortalecerse de campos como la economía, la sociología, la antropología y las ciencias de la cultura en general.

De allí que sea posible pensar que "la contabilidad cultural es un tipo de contabilidad no monetaria que mide y representa el patrimonio cultural de una organización social” (Fuentes \& Peña, 2016, p. 342). Este campo disciplinar proyecta mediciones y valoraciones cualitativas y no monetarias que permitan una mejor representación de la complejidad cultural y sirvan para su protección. Históricamente la contabilidad no ha estado interesada en reconocer, medir, valorar, consolidar, controlar, revelar o representar hechos aislados de la lógica económico-financiera del capital monetario. Sin embargo, los desplazamientos en la preponderancia de la producción industrial y los sectores económicos con campos emergentes como el de la cultura, han generado que esta se interese por controlar y representar sus ejes centrales:

1) la organización económica alrededor del sector cultural, donde la contabilidad tiene un papel central en la reinterpretación de la adaptación del presupuesto, la contabilidad financiera, el control de existencias, la auditoría y la medición monetaria y no monetaria en el campo cultural y creativo como sector de aplicación, y;

2) la representación social y económica de la riqueza generada a partir de la interacción de las comunidades con los elementos derivados de la cultura, las industrias y servicios culturales y creativos (Cano, Arias \& Asuaga, 2020, p. 666).

De esta manera, la contabilidad se transforma en una infraestructura de cálculo y representación de manifestaciones culturales y simbólicas. Es posible y desafiante pero a la vez necesario, generar un modelo de contabilidad multi-valorativa que pueda representar realidades diversas y polifuncionales donde se dé cuenta económica de la realidad cultural.

\section{(Re)pensar una contabilidad para lo cultural y lo creativo}

Una variable explicativa para repensar una contabilidad para lo cultural y lo creativo puede ser el comportamiento económico del sector, que no cumple algunos principios económicos básicos, como la ley de la utilidad marginal decreciente. ${ }^{2}$ Por el contrario, en la cultura se muestra que a más consumo, más demanda. Becker \& Murphy (1988) teorizaron este tema, definiendo que lo que caracteriza al consumo cultural es la "adicción racional", llegando a hacer una analogía con el consumo de drogas. En esta vía, la lógica económica debe ser reinterpretada cuando se trata de analizar la ley de oferta y de demanda asociadas al consumo cultural y el sector creativo, ya que sus patrones de comportamiento en el mercado son muy diferentes de cualquier mercancía económicamente transable, y las métricas disciplinares deben abarcar variables endógenas y exógenas de todos los sectores.

Otro factor está dado en que la disciplina contable y económica se centraba en la producción en serie que se inicia con la revolución industrial. El sector creativo, en cambio, no sólo se trabaja con base en proyectos, ${ }^{3}$ sino que cada producto original es único. No existen dos cuadros iguales, ni dos películas iguales, aun cuando traten el mismo tema. Esto implica dificultades a la hora de valorar los activos, en especial en los museos de arte. Las formas de valuación pueden discrepar dependiendo del contexto económico, el uso social y la representatividad para cada comunidad, y en ello la contabilidad no puede ser miope y sólo asociar los elementos culturales y creativos a una lógica de mercado.

En algunos sectores, tales como el cine, no hay diferenciación de precios para la diversidad cultural. La entrada a los cinemas cuesta lo mismo, así se vea una película costosa o una que insumió menores recursos. El precio cambia por las condiciones materiales para tener contacto con la cultura (sillas, alimentos, sensaciones), 
pero no por la producción cultural (película). Sobre la industria cinematográfica, vale destacar el riesgo y la incertidumbre sobre el éxito de una película. Holywood es la tercera industria mundial de producción de películas, luego de India y Nepal. Su producción anual, por medio de las majors ${ }^{4}$ está en el entorno de las 700 películas (Olaskoaga, 2016). Sin embargo, menos de un 20\% de los films son económicamente rentables (Capitalradio, 2019). Gran parte de la rentabilidad puede apreciarse de forma simbólica, pero no financieramente. Las películas que se convierten en récords de taquillas compensan las pérdidas de otras películas, pero la compañía no sabe cuál película será un éxito y cuál no, descontando excepciones. Estos hechos retan y tensionan al contador y a la contabilidad al momento de elaborar información prospectiva y de planificación, haciendo hincapié en decisiones patrimoniales de reservas. El primer fin de semana de estreno es crucial para el éxito de una película llegando a producir más de un tercio de los ingresos totales de dicha película. Si el fin de semana de estreno no atrae público es probable que salga de cartelera antes del fin de semana siguiente. Por tanto, la contabilidad debe ser dinámica, receptiva a cada día de gestión del cinema, prudencial y no estimativa en el potencial de generar flujos futuros.

Con respecto a las artes escénicas hay un caso que no es común en los procesos productivos, y es la producción de servicios no rivales. Un bien o servicio se define de consumo no rival cuando el consumo de un bien, por un individuo, no impide el consumo simultáneo del mismo bien o servicio por otro individuo. En efecto, varias personas simultáneamente pueden estar disfrutando de un mismo espectáculo, y los ingresos estarán dados por la cantidad de entradas vendidas. Y esto es diferente a los costos, que serán independientes de la cantidad de espectadores. Esto lleva a los contadores a replantearse técnicas básicas como, por ejemplo, la determinación del punto de equilibrio.

Pero no sólo en las artes escénicas se da esta disociación entre ingresos y costos. Otro caso para destacar se da en la radio o en la televisión por aire, en el que deberá analizarse cuál es la figura del cliente. Es claro que ni el oyente radial ni el telespectador está vinculado con las ventas, que son ingresos por publicidad. Nótese la disociación entre las cuentas de ventas y costo de ventas, ya que este último es el costo de producir los programas para los oyentes de una radio o televisión.

Asimismo, surgen nuevos modelos de financiación, como el crowdfunding, que, aunque los aportes individuales pueden no ser relevantes, si la cantidad de donantes es alta, el monto captado puede ser significativo. Los comienzos de estas formas de financiación colectivas pueden retrotraerse a 1997, cuando los fans de un grupo de rock británico, Marillon, colaboraron con US $\$ 60.000$ para financiar una gira por Estados Unidos. El récord de fondos obtenidos bajo esta fuente de financiación se le atribuye al videojuego en línea Star Citizen, que recaudó más de 340 millones de dólares (Arroyo, 2021). Es importante la reflexión contable que deben tener estas donaciones ${ }^{5}$ cuando superan los requerimientos de fondos para la producción. ¿ Pueden considerarse ganancia o deben ser aportes de capital? ¿Si son ganancias, son distribuibles? La contabilidad financiera debe dar respuesta a esta forma no tradicional de financiación que ha sido exitosa en varias partes del mundo, en particular en Estados Unidos y Europa, destacándose dentro de esta última región Inglaterra y los países nórdicos.

Asimismo, la industria creativa está permanentemente innovando y desarrollando nuevos modelos de negocios. En 2017 ocurrió el lanzamiento en nuevo formato de MoviePass, empresa creada por Mitch Lowe, co-fundador de Netflix y socio hasta 2011. El nuevo formato implica que por US $\$ 9,95^{6}$ se pueda concurrir diariamente al cine, en 34.000 salas, que implican el 90\% de las salas de exhibición de Estados Unidos. La compañía asume luego el pasivo con las salas de cine. La forma de financiar este cambio de modelo de negocio ${ }^{7}$ fue asociándose con la firma de datos AOL, quien espera recoger datos de los espectadores, que en un $75 \%$ son milenians (Asuaga, 2011). Cabe cuestionarse el tratamiento contable que se dará a la captación de cada nuevo socio. En los seguros, tanto de autos, incendio, salud, entre otros, la empresa aseguradora asume un riesgo, y un eventual pasivo; asimismo, el asegurado pretende no utilizar el servicio. Sin embargo, esta situación es distinta, ya que el socio de moviepass tiene intención de concurrir al cine más de una vez al mes. Por cada 
vez que concurra, luego de la primera, moviepass obtendría pérdidas y deberá asumir el pasivo que genere cada socio.

Estas formas de negocio no están pensadas, problematizadas ni resueltas en las normas contables, por lo que aún no hay claridad sobre su tratamiento. Pero quizás lo que más cueste incorporar en el modelo económico emergente es la forma no planificada en que se da la producción creativa, comparada con la producción tradicional, tal como puede apreciarse en la figura 2.

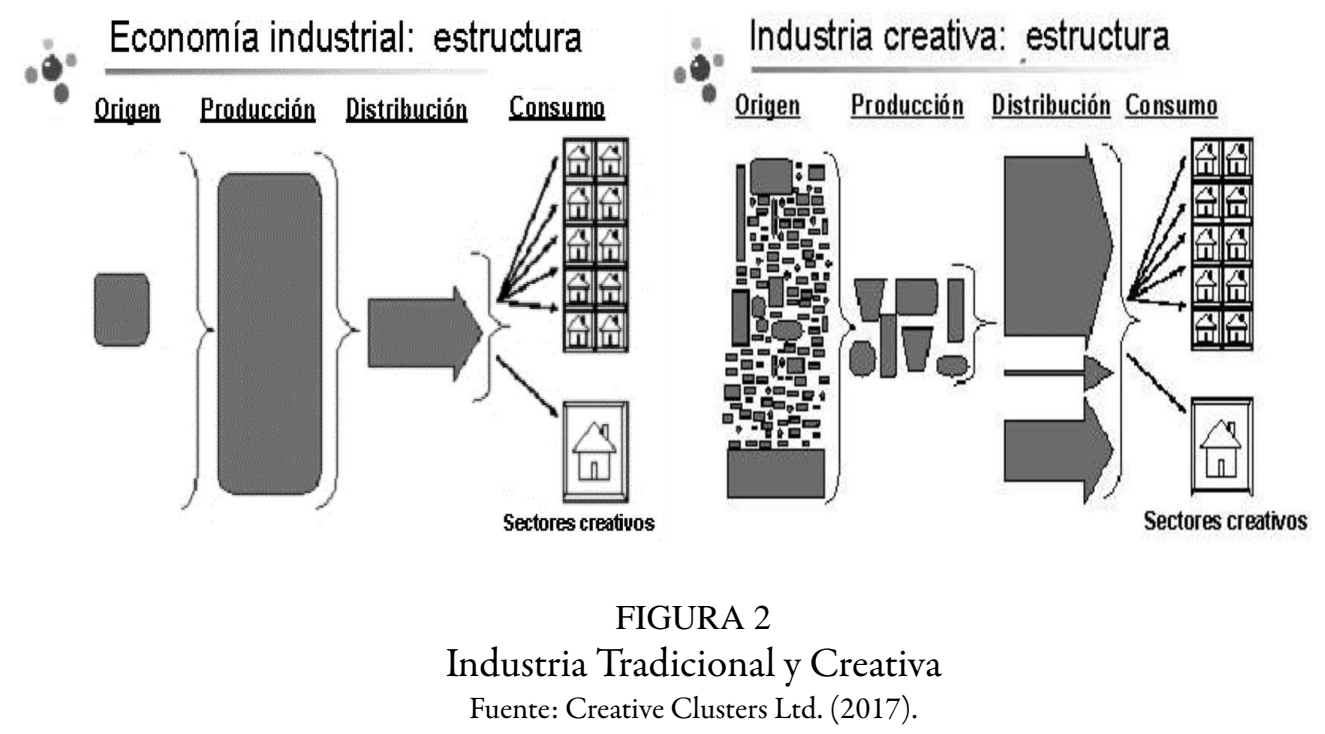

El sector creativo se ha reinventado constantemente buscando nuevos modelos de negocio, nuevas fuentes de financiamiento y creando su propia demanda. La contabilidad deberá dar respuesta a estos cambios, intentando adaptarse al sector creativo y sus peculiaridades.

\section{Consideraciones finales}

$\mathrm{Al}$ reflexionar y analizar lo expuesto es menester auscultar y avanzar en investigaciones que evidencien el potencial que existe entre la denominada economía de la cultura y la contabilidad como cuerpos de conocimiento, ya que tienen varios puntos de conexión en la representación de la realidad socioeconómica de la producción y los servicios derivados de los productos y actividades culturales. A pesar de que los instrumentos de medición y valoración financiera son hegemónicos en la contabilidad tradicional y sus normas asociadas, la economía de la cultura ha dado algunos pasos que invitan al saber contable a expandir su área de aplicación hacia las industrias culturales y creativas. Las universidades no deberían estar omisas ante esta nueva realidad, ya que es la academia la que debe plantearse, en toda su envergadura teórica, el manejo de sus herramientas en un marco conceptual que las sustente.

Este documento realizó una aproximación analítica sobre la importancia de forjar una contabilidad con elementos técnicos, normativos y conceptuales que sean más precisos en la representación y rendición de cuentas de los productos derivados de la economía de la cultura, sus sectores creativos, dinámicos e innovaciones, los cuales se convierten en desafíos académicos, prácticos y jurídicos por dar cuenta de este sector. Una contabilidad multivalorativa, más social que financiera, podría captar formas de crecimiento económico que escapan al concepto clásico de utilidad. La sostenibilidad de muchas nuevas industrias, minoritarias y artesanales que escapan a los indicadores macroeconómicos requieren de estas respuestas para representar su realidad y aportar a la toma de decisiones estratégicas de equilibrio y subsistencia organizacional.

Estos sectores emergentes son susceptibles de todo tipo de procesos de organización, industrialización e inserción en la dinámica societal. Su potencial está centrado en la importancia histórica y antropológica 
de sus manifestaciones, las cuales impactan positivamente a las comunidades y transforman las ecuaciones financieras del capital al proponer nuevas formas y medios de interacción: lo digital, lo intangible, lo particular, lo no-estático o lo relativo al uso social.

La transformación de los modelos de producción y de negocios en formaciones económicas de núcleo cultural, con representaciones simbólicas e históricas, y permeadas por un capital humano creativo, innovador y dinámico, impactan la constitución y puesta en práctica del patrimonio cultural, las artes escénicas y la industria cultural. Por tanto, la economía de la cultura y la contabilidad deben continuar forjando un corpus disciplinar que controle y de cuenta de las nuevas realidades económico-culturales, a través de mecanismos de medición, valoración y control contables que representen una realidad por fuera del tradicional sistema binario, dual y causal de lógica crematística, para abrirle campo a lo complejo, multivalorativo y policausal.

\section{Consideraciones éticas}

El desarrollo del artículo no requirió de ninguna consideración o aval ético

\section{Contribución de los autores}

Los autores participaron equitativamente en la construcción del documento, tanto en su aporte intelectual como material.

\section{Financiación}

No se contó con financiación.

\section{Conflictos de interés}

Los autores declaran que no existe ningún conflicto de interés relacionado con el documento.

\section{Referencias}

Aguado, L., Palma, L., \& Pulido, N. (2017). 50 años de economía de la cultura. Explorando sus raíces en la historia del pensamiento económico. Cuadernos de Economia, 36(70), 197-225. https://doi.org/10.15446/cuad.econ.v 36n70.53813

Anheier, J., Gerhards, J., \& Romo, F. (1995). Forms of capital and social structure in cultural fields: Examining Bourdieu's social topography. American Journal of Sociology, 100(4), 859-903. https://www.jstor.org/stable/27 82154

Araújo, J. (1998). ¿Qué es y qué no es Contabilidad? Lúmina, 2, 101-104. https://doi.org/10.30554/lumina.02.112 6.1998

Arias, J. (2017). Ecología política: desafíos de la contabilidad frente a la justicia ambiental. En-Contexto, 5(6), 303-326. https://ojs.tdea.edu.co/index.php/encontexto/article/view/414

Arias, J., \& Cano, V. (2018). Análisis de la perspectiva transdisciplinar como estrategia pedagógica de enseñanza y de aprendizaje en Contaduría Pública. En C. Pico (Comp.). Experiencias de aprendizaje significativo para la apropiación de conocimientos en ciencias económicas, administrativas y contables. Bogotá: Editorial Politécnico Grancolombiano. 
Arias, J., \& Cano, V. (2020). La contabilidad como reloj socio-organizacional. Perspectiva analítica de la categoría tiempo en contabilidad. Contaduria Universidad de Antioquia, 77, 191-220. https://doi.org/10.17533/udea.r c.n77a07

Arroyo, D. (2021). El crowdfunding de Star Citizen batió su propio récord en 2020 y ya supera los 340 millones. Portal Meristation. https://as.com/meristation/2021/01/04/noticias/1609756270_447501.html

Asuaga, C., \& Esmoris, M. (2006). Indicadores de gestión en organismos públicos. El caso de las orquestas sinfónicas. II Congreso de Costos del Mercosur. Colonia, Uruguay. https://mpra.ub.uni-muenchen.de/105649/

Asuaga, C. (2011). El costo de las obras de arte y la gestión de museos. Madrid: EAE.

Bauman, Z. (2013). Vida líquida. Barcelona: Editorial Austral.

Baumol, W., \& Bowen, W. (1966). Performing Arts-The Economic Dilemma. Cambridge: Twentieth Century Fox.

Becker, G., \& Murphy, K. (1988). A theory of Rational Addiction. The Journal of Political Economy, 96(4), 675-700. https://doi.org/10.1086/261558

Benhamou, F. (2002). Économie du star-system ( $\left.L^{\prime}\right)$. Paris: Odile Jacob.

Benavente, J., \& Grazzi, M. (2017). Impulsando politicas públicas para la creatividad y la innovación. Washington DC: BID.

Banco Interamericano de Desarrollo - BID (2009). Industrias creativas basadas en redes distribuidas Web 2.0. Washington DC: BID.

Banco Interamericano de Desarrollo - BID (2017). Economia Naranja. Innovaciones en América Latina y el Caribe. Washington DC: BID.

Bocella, N., \& Salerno, I. (2016). Creative Economy, Cultural Industries and Local Development. Social and Behavioral Sciences, 223(10), 291-296. https://doi.org/10.1016/j.sbspro.2016.05.370

Bonet, L. (2003). Cultural tourism. In Towse, R. (Ed.). A handbook of cultural economics (chapter 23). London: Edward Elgar Publishing.

Braun, G., \& Rodriguez, R. (2008). Earnings management and accounting values: a test of Gray. Journal of International Accounting Research, 7(2), 1-23. https://doi.org/10.2308/jiar.2008.7.2.1

Buitrago, F., \& Duque, I. (2013). La Economia Naranja. Una oportunidad infinita. Washington DC: BID.

Capitalradio (2019). El cine español, una historia que deja de ser rentable. https://www.capitalradio.es/eventos/cineespanol-rentable_33828547.html

Cano, V. (2018). Elementos conceptuales para valorar el patrimonio cultural: construcción de un corpus necesario. Revista Cientifica General José Maria Córdova, 16(23), 109-124. https://doi.org/10.21830/19006586.301

Cano, V., Arias, J., \& Asuaga, C. (2020). Contabilidad cultural: un campo emergente enfocado en salvaguardar el patrimonio cultural. Revista Cientifica General José Maria Córdova, 18(31), 651-672. http://dx.doi.org/10.21 $830 / 19006586.605$

Carnegie, G., \& Wolnizer, P. (1995). The financial value of cultural, heritage and scientific collections: an Accounting Fiction. Australian Accounting Review, 5(1), 31-47. https://doi.org/10.1111/j.1835-2561.1995.tb00164.x

Carnegie, G., \& Wolnizer, P. (1996). Enabling accountability in museums. Accounting, Auditing, \& Accountability Journal, 9(5). S. 84-99. https://doi.org/10.1108/09513579610151962

Carnegie, G., \& Wolnizer, P. (1999). Unravelling the rhetoric about the financial reporting of public collections as assets. Australian Accounting Review, 9(17). 16-21. https://doi.org/10.1111/j.1835-2561.1999.tb00095.x

Chanchani, S., \& MacGregor, A. (1999). A synthesis of cultural studies in accounting. Journal of Accounting Literature, 18, 1-30. https://www.econbiz.de/Record/a-synthesis-of-cultural-studies-in-accounting-chanchani-shalin/10 006985841

Chenhall, R. (2003). Management control systems design within its organizational context: findings from contingency-based research and directions for the future. Accounting, Organizations and Society, 28(2/3), 127168. https://doi.org/10.1016/S0361-3682(01)00027-7

Cieslewicz, J. K. (2014). Relationships between national economic culture, institutions, and accounting: Implications for IFRS. Critical Perspectives on Accounting, 25(6), 511-528. https://doi.org/10.1016/j.cpa.2013.03.006 
Creative Clusters Ltd. (2017). Industria tradicional y creativa. http://creativeclusters.com/

Drey, P. (1910). Die wirtschaftlichen Grundlagen der Malkunst. Cotta. Berlín.

European Creative Business Network (2015). European creative industries Summit. Ecis Report. Brussels. http://ecbn etwork.eu/wp-content/uploads/2015/09/ECIS-2015-Brussels.pdf

Finlev, T., Maguire, R., Oppenheim, B., \& Skvirsky, S. (2017). El futuro de la economía naranja: Fórmulas creativas para mejorar vidas en América Latina y el Caribe. Washington DC: BID.

Frey, B., \& Stutzer, A. (2002). What can economists learn from happiness research? Journal of Economic Literature, 40(2): 402-435. https://www.jstor.org/stable/2698383

Frey, B., \& Pommerehne, W. (1989). Muses and Markets. Explorations in the Economist of the Arts. Oxford: Blackwell.

Fuentes, J., \& Peña, F. (2016). Un intento de reconstrucción de la contabilidad del chamán Makuna que habita en las selvas del Vaupés en la Amazonia colombiana. Revista Cientifica General José María Córdova, 14(17), 285-346. https://doi.org/10.21830/19006586.16

Fuertes, M., \& Badillo, A. (2016). La dificultad de medir la cultura y la diversidad. Comparación de tres modelos internacionales de medición cultural: MEC-2009, ESSnet-2012 y CAB- 2015, en CIC. Cuadernos de Información y Comunicación, 21, 63-95. https://doi.org/10.5209/CIYC.52878

García-Casella, C. (2012). La persona humana y la ciencia factual cultural aplicada llamada Contabilidad. Teuken Bidikay, 3, 25-36. https://revistas.elpoli.edu.co/index.php/teu/article/view/1060

Gil, J. (2004). Enfoque de la Contabilidad basado en la Tecnologia: un apunte. XV JUC y I JUIC. Buenos Aires: Universidad de Buenos Aires.

Gray, S. (1988). Towards a theory of cultural influence on the development of accounting systems internationally. Abacus, 24(1), 1-15. https://doi.org/10.1111/j.1467-6281.1988.tb00200.x

Gómez, M., \& Ospina, C. M. (2009). Ampliando las fronteras en la disciplina contable: una introducción para la contextualización de los ejemplares heterodoxos. En: Avances interdisciplinarios para una comprensión crítica de la contabilidad. Textos paradigmáticos de las corrientes heterodoxas. Bogotá: Universidad Nacional de Colombia - Universidad de Antioquia.

Gómez, M. (2011). Pensando los fundamentos de la Contabilidad como disciplina académica. Lúmina, 12, 120-150. https://doi.org/10.30554/lumina.12.696.2011

Harrison, G., \& McKinnon, J. (1999). Cross-cultural research in management control systems design: A review of the current state. Accounting, Organizations and Society, 24(5), 483-506. https://doi.org/10.1016/S0361-3682(9 7)00048-2

Herrero, L. (2002). La economía de la cultura en España: una disciplina incipiente. Revista Asturiana de Economía, 23, 147-175. http://www.scielo.org.co/pdf/rei/v12n22/v12n22a6.pdf

Hopwood, A., \& Miller, P. (1994). Accounting as Social and Institutional Practice. London: Cambridge Studies in Managment.

Howkins, J. (2002). The creative economy: How people make money from ideas. London: Penguin.

Jeacle, I. (2012). Accounting and popular culture: framing a research agenda. Accounting, Auditing, \& Accountability Journal, 25(4), 580-601. https://doi.org/10.1108/09513571211225051

Jeacle, I., \& Miller, P. (2016). Accounting, culture, and the state. Critical perspectives on accounting, 37, 1-4. https:/ /doi.org/10.1016/j.cpa.2015.10.001

Kindermann, C. (1903). Volkswirtschaft und Kunst. Jena: Fischer.

Kantis, H., Federico, J., \& Ibarra, S. (2016). Condiciones sistémicas para el emprendimiento dinámico 2016. Novedades $y$ tendencias para fortalecer e integrar los ecosistemas de la región. ICSEd-Prodem.

Luzardo, A., De Jesús, D., \& Perez, M. (2017). Economia naranja: Innovaciones que no sabias que eran de América Latina y el Caribe. Washington DC: BID.

Mautz, R. (1988). Monuments, mistakes and opportunities. Accounting Horizons, 2(2), 123-128.

Micallef, F., \& Peirson, G. (1997). Financial reporting of cultural, heritage, scientific and community collections. Australian Accounting Review, 7(13).31-37. https://doi.org/10.1111/j.1835-2561.1997.tb00025.x 
Miller, P. (1998). The margins of accounting. European Accounting Review, 7(4), 605-621. https://doi.org/10.1080/ 096381898336213

Mossetto, G. (2013). Aesthetics and economics. Springer Science, \& Business Media.

Olaskoaga, A. (2016). El mercado del cine en EEUU. Estudios de Mercado. Los Ángeles.

Ospina, C. (2006). Sobre la investigación en contabilidad. Algunos apuntes. Porik An, 11, 73-121. http://www.unica uca.edu.co/porik_an/imagenes_3noanteriores/No.11porikan/articulo2.pdf

Palma, L., \& Aguado, L. (2010). Economía de la cultura. Una nueva área de especialización de la economía. Revista de Economia Institucional, 12(22), 129-165. https://revistas.uexternado.edu.co/index.php/ecoins/article/view 1386

Potter, B. (2005). Accounting as social and institutional practice: perspectives to enrich our understanding of accounting change. Abacus, 41(3), 265-289. https://doi.org/10.1111/j.1467-6281.2005.00182.x

Pratt, A. (1997). The cultural industries production system: A case study of employment change in Britain, 1984-91. Environment and planning A, 29(11), 1953-1974. https://doi.org/10.1068/a291953

Pratt, A., \& Jeffcutt, P. (2011). Creativity, innovation and the cultural economy. London, \& New York: Routledge.

Quartesan, A., Romis, M, \& Lanzafam, L. (2007). Las industrias culturales en América Latina y el Caribe: desafios y oportunidades. Washington DC: BID.

Rausell-Köster, P. (1999). Politicas y sectores culturales en la Comunidad Valenciana. Valencia: Tirant lo Blanch.

Rausell-Köster, P. (2017). Culture, Creativity and Economic Progress. In Mickov, B., \& Doyle, J. (Eds.). Culture, Innovation and the Economy. Routledge

Rueda, G. (2002). Desarrollo alternativo y contabilidad pública: hacia una línea de investigación. Revista Nómadas, 16, 195-203. http://nomadas.ucentral.edu.co/nomadas/pdf/nomadas_16/16_15R_Desarrolloalternativoycon tabilidad.pdf

Sá, A. (1995). Autonomía y calidad científica de la contabilidad. Revista de la Facultad de Contaduría Pública, 17.

Sarmiento, H. (2007). Retorno a la idea. Elementos metodológicos para la construcción de conceptos propedéuticos de investigación contable. Lúmina, 8, 43-57. https://doi.org/10.30554/lumina.08.1184.2007

Shorthose, J. (2004). Accounting for independent creativity in the new cultural economy. Media International Australia Incorporating Culture and Policy, 112(1), 150-161. https://doi.org/10.1177/1329878X0411200112

Smith, A. (1776). La riqueza de las naciones, edición consultada de 2009. Madrid: Prisa Innova S.L.

Throsby, D., \& Withers, G. (1979). The Economist of the Performing Arts. Londres: Arnold.

Throsby, D. (1994). The production and consumption of the arts: a view of cultural economist. Journal of Economic Literature, 32(1), 1-29. https://www.jstor.org/stable/2728421

UNESCO (2013). Industrias Creativas. http://www.unesco.org/new/en/culture/themes/creativity/creative-industr ies/

UNESCO, \& PNUD (2014). Informe sobre la Economía Creativa. Edición especial 2013. One United Nations Plaza, New York. http://www.unesco.org/culture/pdf/creative-economy-report-2013-es.pdf

Zan, L, Blackstock, A., Cerutti, G., \& Mayer, C. (2000). Accounting for art. Scandinavian Journal of Management, 16, 335-347. https://doi.org/10.1016/S0956-5221(00)00006-3

\section{Notas}

* Artículo de reflexión

1 En Colombia, los virajes industriales hacia lo cultural y creativo se conocen como 'economía naranja', que de acuerdo con la noción de Buitrago \& Duque (2013) y posteriormente reforzado por el BID (2017), contempla un sector económico renovado centrado en el talento, la creatividad, la conectividad, el rescate de la propiedad intelectual y los derechos de autor, y el legado cultural regional. Empero, debe recalcarse que la perspectiva de Buitrago \& Duque (2013) es una apropiación de las ideas fundantes de Howkins (2002). 
2 El ejemplo clásico, es que, ante una sensación de sed, el primer vaso de agua proporciona utilidad a quien lo consume, pero esa utilidad es decreciente a medida que se consumen más vasos de agua.

3 Por ejemplo, una obra de teatro se compone de ensayos, estreno/lanzamiento, explotación y giras, teniendo una vida útil acotada. Es interesante el cuestionamiento sobre los postulados e hipótesis de empresa en marcha aceptados por la doctrina contable.

4 Los majors son los sies conglomerados que acaparan un poco más del 80\% de los ingresos de taquilla en Estados Unidos y Canadá. Componen este grupo, también llamado "the big six" Columbia, Paramount, Disney, 20th Century Fox, Universal Pictures y Warner Bros. Según Buitrago y Duque (2013), si se consideran todos los formatos y se incluye la producción de Bollywood en India y Nollywood en Nigeria, la producción supera las 4.000 películas anuales.

5 Aunque en sus comienzos se trataba exclusivamente de donaciones, hoy en día también se utiliza el crowdfunding como como mecanismo de intermediación financiera en sectores no-creativos, apareciendo el crowdfunding con retorno financiero, en donde el inversor participa del negocio o es acreedor del mismo, recibiendo intereses periódicos hasta el saldo de la deuda

6 El ingreso a cinemas en Estados Unidos tiene un costo entre US\$ 9 y US\$ 10, aproximadamente.

7 Antes moviepass cobraba US\$29,95 mensual y existían ciertas restricciones.

\section{Licencia Creative Commons CC BY 4.0}

Sugerencia de citación:: Arias, J., Asuaga, C., \& Cano, V. (2021). Contabilidad, economía de la cultura y sectores creativos: necesidades y desafíos de una vinculación técnica y disciplinar. Cuadernos de Contabilidad, 22. https://doi.org/10.11144/Javeriana.cc22.cecs 